\title{
Preoperative Diagnosis of Ovarian Pregnancy by Transvaginal Ultrasound: A Case Report
}

\author{
Lijuan Sun, MD ${ }^{a}$, Hui Li, MS ${ }^{b}$, Juhong Liu, MS ${ }^{b}$, Cuixia Guo, MS ${ }^{a}$, Qingqing Wu MD ${ }^{a, *}$ \\ ${ }^{a}$ Department of Ultrasound, Beijing Obstetrics and Gynecology Hospital, Capital Medical University, Beijing, China; ${ }^{b}$ Department of \\ Gynecology, Beijing Obstetrics and Gynecology Hospital, Capital Medical University, Beijing, China \\ Received March 14, 2020; revision received March 26, 2020; accepted May 10, 2020
}

\begin{abstract}
Primary ovarian pregnancy is rare in the classifications of ectopic pregnancy with an incidence of $0.5-3 \%$ of all ectopic gestations. The surgical management of ovarian pregnancy is different from the operation of salpingocyesis. Preoperative diagnosis in early pregnancy can help to guide the optimal management. We have reported the case of a 37- year-old woman who presented with a naturally conceived ovarian pregnancy. Transvaginal ultrasound was used in this patient, and made the correct diagnosis of ovarian pregnancy before surgery. We found that transvaginal ultrasound can be helpful in the diagnosis of the location of ectopic pregnancies.
\end{abstract}

Key words: Ovarian pregnancy; Transvaginal ultrasound; Preoperative diagnosis

Advanced Ultrasound in Diagnosis and Therapy 2021;01:051-053

DOI: 10.37015/AUDT.2021.200015

$\mathrm{P}$ rimary ovarian pregnancy is rare in the classifications of ectopic pregnancy with an incidence of $0.5-3 \%$ of all ectopic gestations [1]. The surgical management of ovarian pregnancy is different from the operation of salpingocyesis. Preoperative diagnosis in early pregnancy can help to guide the optimal management. We have reported the case of a 37- year-old woman with ovarian pregnancy that was accurately diagnosed by transvaginal ultrasound preoperatively.

\section{Case report}

A 37-year-old gravida 3 and para 1 woman was admitted because of 40 days of amenorrhea and vaginal bleeding for 4 days. The patient had conceived naturally, and suffered from no other diseases besides anemia. The vital signs of the woman were stable, and there were no positive findings in physical examination.

A pregnancy test was positive after 37 days of amenorrhea. Transvaginal ultrasound could not locate a gestational sac in the uterus, but revealed a $1.8 \times 1.8 \times 1.7$ $\mathrm{cm}$ mass with cyst-solid component located around the corpus luteum. The color Doppler flow imaging (CDFI) showed there was a small amount of blood flow around the mass, and the relationship between the mass and the corpus luteum was close (Fig. 1 and Fig. 2). The small embryo bud measuring $1 \mathrm{~mm}$ in length and fetal heart beat were observed inside the mass. A small amount of pelvic effusion was seen behind the uterus. In addition, multiple hysteromyomas were demonstrated, and the largest one $(5.6 \times 4.3 \times 5.0 \mathrm{~cm})$ located in the anterior wall of the uterus. The result of blood testing showed that human chorionic gonadotropin (HCG) was 3688.62 $\mathrm{IU} / \mathrm{L}$ and progesterone was $10.00 \mathrm{ng} / \mathrm{ml}$.

The diagnosis of ectopic pregnancy and hysteromyomas were clear combined with the ultrasound findings and the result of the blood testing. We suspected the ovarian pregnancy according to the ultrasound features, a gestational sac around the corpus luteum and the close relationship between them. Ultimately, the patient underwent the laparoscopic surgery with the resection of ovarian pregnancy and some ovarian tissue. The left ovarian pregnancy and hysteromyomas were confirmed by surgical findings and pathological results (Fig. 3).

\footnotetext{
* Corresponding author: Department of Ultrasound, Beijing Obstetrics and Gynecology Hospital, Capital Medical University, 251 Yaojia Yuan Road, Chaoyang District, Beijing 100026, China

e-mail:wuqq2007@163.com unrestricted use, distribution and reproduction in any medium provided that the original work is properly attributed.
} 


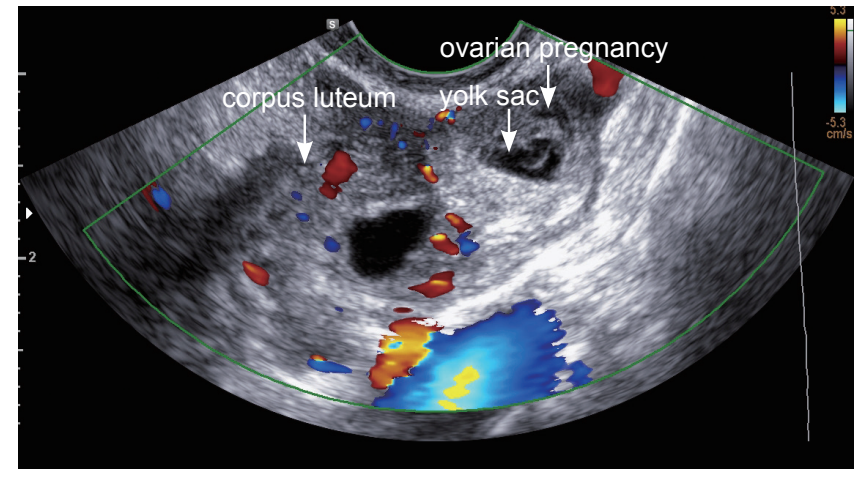

Figure 1 The cyst-solid mass with $1.8 \times 1.8 \times 1.7 \mathrm{~cm}$ in size located around the corpus luteum, and the relationship between the mass and the corpus luteum was close by transvaginal ultrasound. The yolk sac was inside the mass.

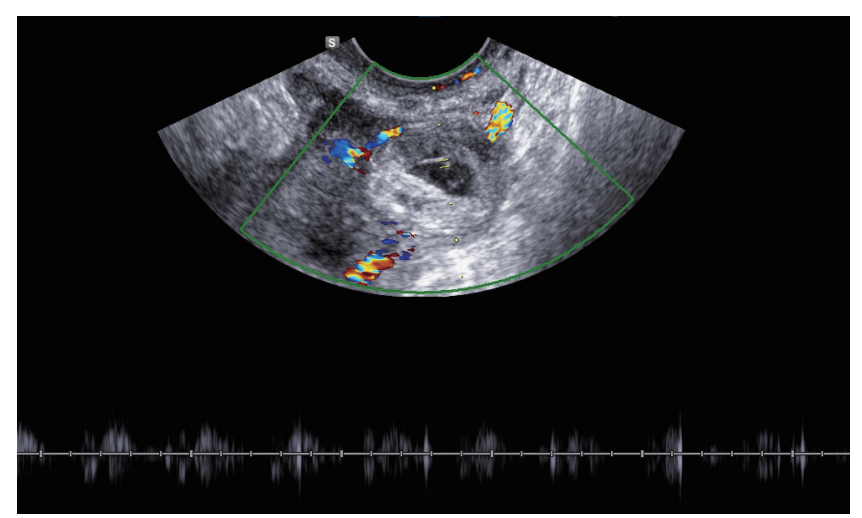

Figure 2 Fetal heart beat was observed inside the mass by transvaginal ultrasound.

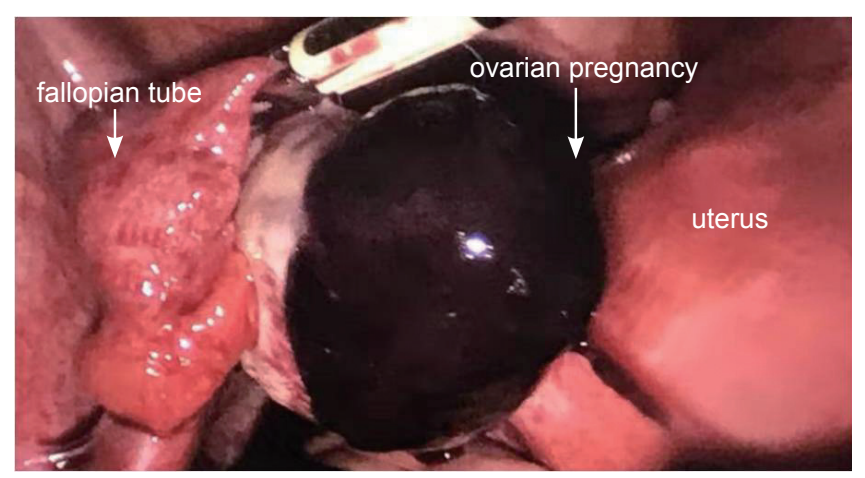

Figure 3 The findings of laparoscopic surgery showed the gestational sac (arrow) located inside the left ovary, and the fallopian tube seemed to be normal. The left ovarian pregnancy was confirmed by the findings of laparoscopic surgery.

\section{Discussion}

Owing to the low incidence of ovarian pregnancy, most studies are case reports. The underlying mechanism of ovarian pregnancy was considered implantation within the ovary after fertilization occurring outside the tube, or the failure of ovulation $[2,3]$. The major risk factors for ovarian pregnancy include the use of IUD, pelvic inflammatory disease, assisted reproductive technology, and previous ectopic pregnancy or endometriosis and so on $[1,4]$. However, the patient in our study had no related risk factors. It has been reported that $39.5 \%$ to $50 \%$ of women had no risk factors [5].

The classic clinical symptoms of ovarian pregnancy include amenorrhea, abdominal pain, and vaginal bleeding, which are similar to the other kinds of ectopic pregnancy [6]. Some cases had no specific clinical manifestations prior to rupture. Therefore, it is difficult to diagnosis ovarian pregnancy preoperatively according to only the clinical symptoms. Most of the diagnosis for ovarian pregnancy depends on the histopathological findings $[7,8]$.

To the best of our knowledge, tubal pregnancy is the most common type of ectopic pregnancy, and the management is different from ovarian pregnancy. Therefore, it is very important for the correct preoperative differential diagnosis. In fact, distinguishing tubal pregnancy from ovarian pregnancy by imaging examination is very difficult as a result of the close distance between fallopian tube and ovary. With the advancement of transvaginal ultrasound, it is possible for the preoperative diagnosis of ovarian pregnancy with high resolution. The boundary between the mass of ectopic pregnancy and ovary can be demonstrated by transvaginal ultrasound. Meanwhile, the differential diagnosis between corpus luteum and the mass of ovarian pregnancy can be showed, and the latter is characterized by hyperechoic ring or mass [9]. However, preoperative diagnosis of ovarian pregnancy is still a challenge.

We summarized the features of ovarian pregnancy differing from other adnexal masses on transvaginal ultrasound: First, a definite mass like gestational sac is found in the adnexa. Identification of an embryo or a yolk sac is quite rare because most of these pregnancies have a low gestational age; however, when present, the diagnostic accuracy of ultrasound will be increased. Comstock et al. [10] discovered a wide echogenic ring with a small internal echo lucent area as the most frequent ultrasound feature of gestational sac. Second, the relationship between the suspicious mass and the ovary is very close, and there is no boundary between them. This feature is very critical for distinguishing it from tubal pregnancy. However, if the woman has a history of pelvic surgery or adhesions in the pelvic cavity, an unclear border between fallopian tube and ovary could also be observed. Spiegelberg has recommended four criteria for the diagnosis of primary ovarian pregnancy: (1) An intact fallopian tube with the fimbriae separate from the ovary; (2) The gestational sac should be located in the normal position of the ovary; (3) The gestational sac should be connected to the uterus with utero-ovarian ligament; (4) Ovarian tissue is around the gestational sac. Last, though the imaging characteristics of ruptured ovarian 
pregnancy and the rupture of corpus luteum are similar, the abnormal level of blood HCG (human chorionic gonadotropin) demonstrates the strong evidence which supports the diagnosis of ovarian pregnancy.

\section{Conclusion}

We have presented a rare case of a 37-year-old female patient with a left ovarian pregnancy, and have described the features of sonography, which will be helpful in the diagnosis of ovarian pregnancy.

\section{Acknowledgement}

This research was supported by Beijing Municipal Administration of Hospitals' Ascent Plan, Code: DFL20151302.

\section{Conflict of Interest}

The authors declare that they have no conflict of interest.

\section{References}

[1] Goyal LD, Tondon R, Goel P, Sehgal A. Ovarian ectopic pregnancy: A 10 years' experience and review of literature. Iran J Reprod Med
2014; 12: 825-30.

[2] Ferland RJ, Chadwick DA, O'Brien JA, Granai CO 3rd. An ectopic pregnancy in the upper retroperitoneum following in vitro fertilization and embryo transfer. Obstet Gynecol 1991; 78: 544-6.

[3] Feit H, Leibovitz Z, Kerner R, Keidar R, Sagiv R. Ovarian pregnancy following in vitro fertilization in a woman after bilateral salpingectomy: a case report and review of the literature. J Minim Invasive Gynecol 2015; 22: 675-77.

[4] Shan N, Dong D, Deng W, Fu Y. Unusual ectopic pregnancies: a retrospective analysis of 65 cases. J Obstet Gynaecol Res 2014; 40: $147-54$.

[5] Shah N, Khan NH. Ectopic pregnancy: presentation and risk factors. $J$ Coll Phys Surg Pak 2005; 15: 535-38.

[6] Robertson JJ, Long B, Koyfman A. Emergency medicine myths: ectopic pregnancy evaluation, risk factors, and presentation. J Emerg Med 2017; 53: 819-28.

[7] Hallatt JG. Primary ovarian pregnancy: a report of twenty-five cases. Am J ObstetGynecol 1982; 143: 55-60.

[8] Choi HJ, Im KS, Jung HJ, Lim KT, Mok JE, Kwon YS. Clinical analysis of ovarian pregnancy: a report of 49 cases. Eur J Obstet Gynecol Reprod Biol 2011; 158: 87-9.

[9] Ge L, Sun W, Wang L, Cheng L, Geng C, Song Q, et al. Ultrasound classification and clinical analysis of ovarian pregnancy: a study of 12 cases. J Gynecol Obstet Hum Reprod 2019; 48: 731-37.

[10] Comstock C, Huston K, Lee W. The ultrasonographic appearance of ovarian ectopic pregnancies. Obstet Gynecol 2005; 105: 42-5. 